\title{
A low-carbohydrate ketogenic diet induces the expression of very-low-density lipoprotein receptor in liver and affects its associated metabolic abnormalities
}

Tetsuya Okuda (iD) ${ }^{1 *}$

\begin{abstract}
A low-carbohydrate ketogenic diet (LCKD) promotes the progression of hepatic steatosis in C57BL/6 wild-type mice, but improves the condition in leptin-deficient obese $(o b / o b)$ mice. Here, we show a novel effect of LCKD associated with the conflicting effects on these mice. Gene expression microarray analyses showed that expression of the VIdlr gene, which encodes the very-low-density lipoprotein receptor (VLDLR), was induced in LCKD-fed ob/ob mice. Although the VLDLR is not normally expressed in the liver, the LCKD led to VLDLR expression in both ob/ob and wild-type mice. To clarify this effect on VLDL dynamics, we analyzed the lipid content of serum lipoproteins and found a marked decrease in VLDL-triglycerides only in LCKD-fed wild-type mice. Further analyses suggested that transport of triglycerides via VLDL from the liver to extrahepatic tissues was inhibited by LCKD-induced hepatic VLDLR expression, but rescued under conditions of leptin deficiency.
\end{abstract}

npj Science of Food (2019)3:25

; https://doi.org/10.1038/s41538-019-0058-4

\section{INTRODUCTION}

Consumption of low-carbohydrate ketogenic diets (LCKDs) has been shown to have diverse beneficial effects in promoting health and alleviating pathologic conditions. For example, LCKDs exhibit antiepileptic effects, ${ }^{1}$ promote weight loss in obesity, ${ }^{2}$ and enhance control of type 2 diabetes mellitus. ${ }^{3}$ LCKDs also reportedly have beneficial effects in patients with autism, ${ }^{4}$ cardiomyopathy, ${ }^{5}$ neurodegenerative diseases, ${ }^{1}$ and cancer. ${ }^{6}$ The effective and safe use of LCKDs based on scientific evidence has contributed to both health promotion and disease treatment; however, the molecular mechanisms underlying the beneficial effects of LCKDs in vivo remain largely unknown.

As a clinical trial demonstrated that restricting carbohydrate intake can reduce intrahepatic triglyceride levels, ${ }^{7}$ increased research attention has focused on the use of LCKDs in treating liver steatosis. ${ }^{8}$ However, children with epilepsy have been shown to develop liver steatosis with long-term consumption of a LCKD; ${ }^{9}$ thus, careful investigation of this effect is needed. A number of studies using rodent models have analyzed the role of LCKDs in the development of liver steatosis. ${ }^{10-16}$ Most of these studies used a common LCKD for rodents (Bio-Serv F3666), ${ }^{10-13,15,16}$ with subsequent development of steatosis observed in the liver of the LCKD-fed mice. ${ }^{10-12,16}$ However, surprisingly, we found that the F3666 diet had a dramatic preventative effect on the progression of liver steatosis in juvenile leptin-deficient obese $(o b / o b)$ mice. ${ }^{13}$ We found that the underlying mechanism involves suppression of de novo triglyceride synthesis. ${ }^{13,16}$ This LCKD also suppresses de novo triglyceride synthesis in C57BL/6J (wild-type) mice that develop liver steatosis. ${ }^{10-12,16}$ These conflicting results suggest that the beneficial effect of the LCKD on liver steatosis in $o b / o b$ mice is regulated by an as yet unknown mechanism that is nonfunctional in wild-type mice.

To clarify the underlying mechanism of these effects, we analyzed several molecular parameters in the liver of LCKD-fed ob/ $o b$ mice and found a novel effect of the LCKD in up-regulating expression of the very-low-density lipoprotein receptor (VLDLR) in the liver. Although this receptor is expressed only minimally in the liver, ${ }^{17}$ LCKD feeding significantly up-regulated VLDLR expression in the liver of $o b / o b$ and wild-type mice. Further analyses showed that VLDL-triglyceride levels were significantly lower in the serum of LCKD-fed wild-type mice. In addition, serum activity of lipoprotein lipase (LPL), which mediates triglyceride uptake from VLDL into extrahepatic tissues, ${ }^{18}$ was also down-regulated. In contrast, marked release of VLDL-triglycerides was observed in LCKD-fed ob/ob mice, whereas LPL activity was maintained. These alterations in both strains were correlated with the conflicting liver steatosis phenotypes.

The results of our study suggest that the transport of triglycerides via VLDL from the liver to extrahepatic tissues is inhibited by LCKD-induced hepatic VLDLR up-regulation under low LPL activity, whereas this inhibition of triglyceride transport is rescued under conditions of leptin deficiency.

\section{RESULTS}

Characterization of liver phenotypes in LCKD-fed mice

Wild-type and $o b / o b$ mice of the inbred strain C57BL/6J were used in this study and fed the Bio-Serv F3666 LCKD. F3666 is a very-lowcarbohydrate, low-protein, high-fat ketogenic diet developed to induce efficient production of ketone bodies in rodents. ${ }^{19}$ In ob/ob mutant mice fed a diet of regular chow, hyperglycemia typically develops at $\sim 10$ weeks of age. ${ }^{20}$ Our initial goal was to reverse this phenotype via feeding mice the LCKD and analyze the diet's effects on tissues at the molecular level. The mice were fed the LCKD over the period 5-12 weeks of age in the dietary experiment. $^{13}$ We found that the LCKD feeding effectively reversed the hyperglycemic phenotype in female ob/ob mice during this period and therefore employed these conditions in subsequent experiments. ${ }^{13}$ The average blood glucose levels during the experimental period were as follows: chow-fed ob/ob mice, $194.30 \pm 43.78 \mathrm{mg} \mathrm{dl}^{-1}$; LCKD-fed ob/ob mice, $106.96 \pm$ $26.41 \mathrm{mg} \mathrm{dl}^{-1}$; chow-fed wild-type mice, $154.08 \pm 20.55 \mathrm{mg} \mathrm{dl}^{-1}$;

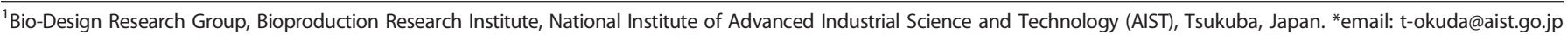



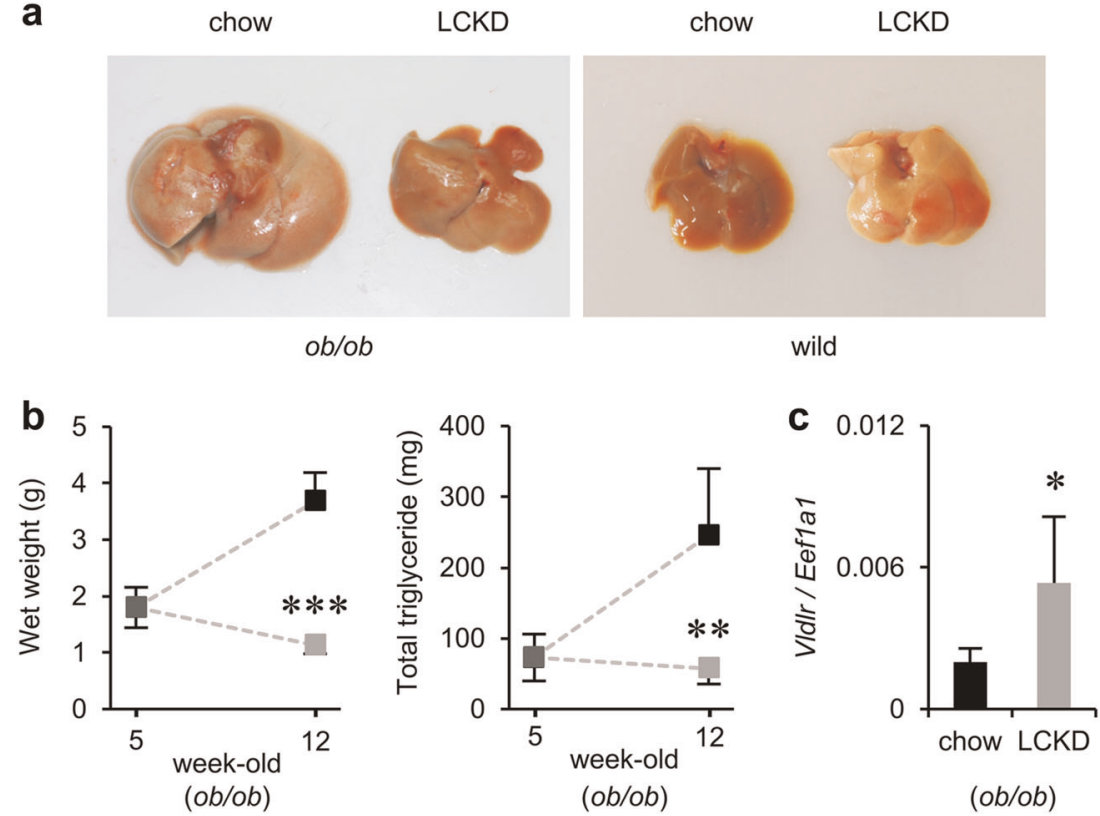

Fig. 1 Effect of LCKD feeding on liver phenotypes. a Morphology of the liver after 7 weeks of feeding. $\mathbf{b}$ Wet weight $(n=11-13)$ and total triglyceride levels $(n=5-6)$ in the liver. c Real-time PCR analysis of expression of the VIdlr gene $(n=6)$. The relative expression level is shown as the ratio relative to expression of the internal standard (Eef1a1). Statistical significance was assessed using the two-tailed Student's $t$ test. ${ }^{*} P<$ $0.05,{ }^{* *} P<0.01,{ }^{* *} P<0.001$, chow-fed vs. LCKD-fed. Black squares and bars, regular chow-fed mice; gray squares and bars, LCKD-fed mice. Mean \pm SD

and LCKD-fed wild-type mice, $114.30 \pm 15.05 \mathrm{mg} \mathrm{dl}^{-1}$. Production of ketone bodies ( $\beta$-hydroxybutyrate) was observed in both the $o b / o b$ and wild-type mice. ${ }^{13,16,21}$

Figure 1a shows the morphology of the liver after 7 weeks of LCKD feeding. In ob/ob mice, regular chow promoted significant steatosis associated with enlargement of the liver (left panel). Liver weight and total amount of triglycerides increased by more than 2-fold compared to values prior to the start of the experiment (Fig. 1b). The LCKD is known to inhibit the progression of liver steatosis in $o b / o b$ mice. ${ }^{13}$ Compared to chow-fed mice, the liver weight and total amount of triglycerides decreased by at least $70 \%$ in LCKD-fed mice (Fig. 1b). The average triglycerides $(\mathrm{mg})$ to liver weight $(\mathrm{g})$ ratios were as follows: chow-fed ob/ob mice, $65.75 \pm 24.7 \mathrm{mg} \mathrm{g}^{-1}$; and LCKDfed ob/ob mice, $48.79 \pm 16.93 \mathrm{mg} \mathrm{g}^{-1}$. In contrast, the LCKD strongly promoted steatosis in wild-type mice. In LCKD-fed wildtype mice, the total amount of triglycerides in the liver has been shown to increase by more than 3 -fold. ${ }^{16}$ Although liver weight remained unchanged in the present study, the organ became discolored as a result of excessive triglyceride accumulation (Fig. 1a, right panel). The average triglycerides ( $\mathrm{mg})$ to liver weight $(\mathrm{g})$ ratios were as follows: chow-fed wild-type mice, $19.17 \pm 5.68 \mathrm{mg} \mathrm{g}^{-1} ;$ and LCKD-fed wild-type mice, 63.65 \pm $16.44 \mathrm{mg} \mathrm{g}^{-1}$. In both strains, body weight gain and diet intake (kcal per day) were similar in the chow- and LCKD-fed groups during the experimental period. ${ }^{13,16}$ Although LCKD feeding improved the steatosis associated with enlargement of the liver, LCKD-fed ob/ob mice became obese to the same degree as chow-fed $o b / o b$ mice. ${ }^{13}$ The average final body weights were as follows: chow-fed ob/ob mice, $53.16 \pm 2.45$ g; LCKD-fed ob/ob mice, $52.47 \pm 3.45 \mathrm{~g}$; chow-fed wild-type mice, $19.16 \pm 1.79 \mathrm{~g}$; and LCKD-fed wild-type mice, $17.87 \pm 2.02 \mathrm{~g}$. The average caloric intake during the experimental period was as follows: chow-fed ob/ob mice, $18.56 \pm 0.4 \mathrm{kcal}$ per day; LCKD-fed ob/ob mice, $17.27 \pm 2.43 \mathrm{kcal}$ per day; chow-fed wild-type mice, $10.48 \pm$ $0.46 \mathrm{kcal}$ per day; and LCKD-fed wild-type mice, $10.41 \pm 1.5 \mathrm{kcal}$ per day.

\section{LCKD effect on hepatic gene expression}

A gene expression microarray analysis was conducted to elucidate the molecular mechanism underlying the improvement of liver steatosis in LCKD-fed ob/ob mice. Data for all genes detected as specific signals were compared with data for mice fed the regular chow $(n=3)$ and deposited in the NCBI Gene Expression Omnibus (GEO; accession number GSE115342). The overall features of the results have been published elsewhere. ${ }^{21}$

A novel result observed in the present study was significant upregulation of the VIdlr gene, which encodes the VLDLR. The microarray analysis revealed significant up-regulation $\left(\log ^{2}\right.$ ratio [LCKD/chow] of $+1.78 ; P<0.05)$ of VIdlr, which was confirmed in validation experiments using real-time PCR (Fig. 1c) with a greater number of samples $(n=6)$. No apparent effects on other factors associated with VLDL metabolism were observed in this experiment.

Effect of the LCKD on induction of VLDLR in the liver As VLDL regulates the release of triglycerides from the liver to the blood stream, ${ }^{18}$ we expected that up-regulation of VIdlr would correlate with the liver phenotype in LCKD-fed mice. Immunoblot analysis showed that VLDLR, which is not normally expressed in the liver, ${ }^{17,22}$ was detected in the liver of mice fed the LCKD (Fig. 2). However, the induction of VLDLR expression was observed in both $o b / o b$ (Fig. 2a) and wild-type (Fig. 2b) mice, indicating that this effect is not specific to $o b / o b$ mice. Although individual differences were observed, further statistical analyses indicated that the effect was associated with the LCKD (Fig. 2c). The statistical analyses also revealed that the level of VLDLR expression in LCKD-fed wild-type mice was higher than that in ob/ob mice $(P<0.01)$.

Characterization of lipid content of lipoproteins and LPL activity in serum

To clarify the effect of hepatic VLDLR on lipoprotein dynamics, we subsequently analyzed the lipid content of serum lipoproteins (Table 1). We found that levels of VLDL-triglycerides were 
a

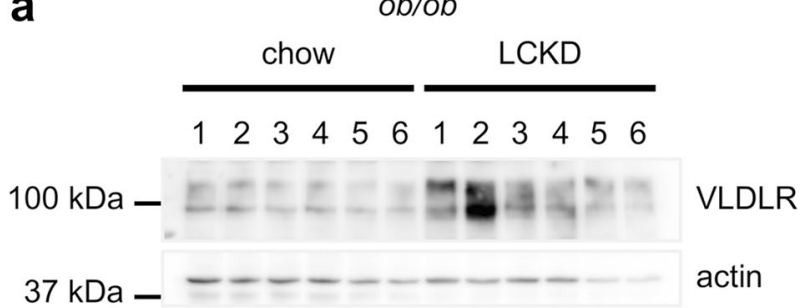

b wild

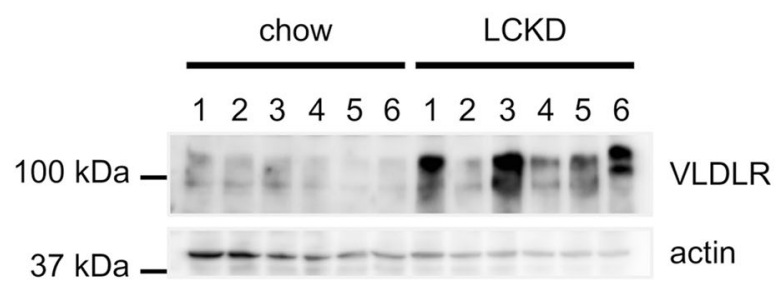

C

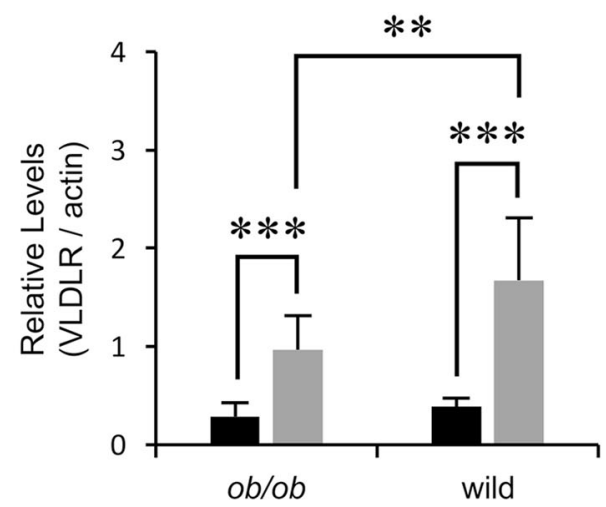

Fig. 2 Immunoblot analysis of VLDLR in the liver. Representative images of immunoblotting of VLDLR in the liver of individual $o b / o b$ a or wild-type $\mathbf{b}$ mice $(n=6)$. Actin is shown as an internal standard. Band intensities were analyzed as previously reported $^{16}$ to determine relative levels (c, VLDLR/actin). ob/ob, $n=16-17$; wildtype, $n=6$. Statistical significance was assessed using the two-tailed Student's $t$ test. ${ }^{*} P<0.01,{ }^{* *} P<0.001$. Black squares and bars, regular chow-fed mice; gray squares and bars, LCKD-fed mice. Mean $\pm \mathrm{SD}$

markedly lower in LCKD-fed wild-type mice, but unchanged in LCKD-fed ob/ob mice. In contrast, VLDL-cholesterol levels were unchanged in LCKD-fed wild-type mice, but significantly lower in LCKD-fed $o b / o b$ mice. These results indicate that the release of VLDL from the liver to the blood stream is not impaired in either LCKD-fed mouse strain; rather, only VLDL-mediated triglyceride release is specifically inhibited in LCKD-fed wild-type mice. The increase in LDL-cholesterol levels in LCKD-fed wild-type mice also supports the normal release of VLDL from the liver in these mice. In LCKD-fed ob/ob mice, the cholesterol content of the VLDL fraction was considerably lower than the triglyceride content, indicating that triglyceride-rich VLDL was generated in these mice. Furthermore, LDL-triglyceride levels were significantly higher in LCKD-fed ob/ob mice, but unchanged in LCKD-fed wild-type mice, which indicates a difference between $o b / o b$ and wild-type mice in terms of conversion from VLDL-triglycerides to LDL-triglycerides. As this process is mediated by $L P L,{ }^{18}$ we preliminarily measured the activity of LPL in the serum and found a significant decrease only in LCKD-fed wild-type mice (Supplementary Fig. S1). In contrast, serum LPL activity was the same in LCKD- and regular chow-fed ob/ob mice, indicating that the LCKD-associated
Table 1. Serum lipoprotein lipid content

\begin{tabular}{|c|c|c|c|c|}
\hline \multirow[b]{3}{*}{ Diet } & \multicolumn{4}{|l|}{ Strain } \\
\hline & \multicolumn{2}{|l|}{$o b / o b$} & \multicolumn{2}{|l|}{ Wild type } \\
\hline & Chow & LCKD & Chow & LCKD \\
\hline \multicolumn{5}{|l|}{ Parameters } \\
\hline \multicolumn{5}{|c|}{ Triglyceride $\left(\mathrm{mmol} \mathrm{I}^{-1}\right)$} \\
\hline Total & $1.23 \pm 0.22$ & $1.47 \pm 0.30$ & $1.30 \pm 0.20$ & $0.18 \pm 0.03^{* *}$ \\
\hline $\mathrm{CM}$ & $0.31 \pm 0.06$ & $0.08 \pm 0.02^{*}$ & $0.14 \pm 0.03$ & $0.02 \pm 0.005^{* *}$ \\
\hline VLDL & $0.58 \pm 0.11$ & $0.53 \pm 0.14$ & $0.97 \pm 0.15$ & $0.04 \pm 0.01^{* *}$ \\
\hline LDL & $0.23 \pm 0.04$ & $0.65 \pm 0.17^{* * *}$ & $0.15 \pm 0.02$ & $0.10 \pm 0.01$ \\
\hline $\mathrm{HDL}$ & $0.08 \pm 0.02$ & $0.07 \pm 0.02$ & $0.04 \pm 0.01$ & $0.02 \pm 0.002^{*}$ \\
\hline \multicolumn{5}{|c|}{ Cholesterol $\left(\mathrm{mmoll}^{-1}\right)$} \\
\hline Total & $4.08 \pm 0.28$ & $5.66 \pm 0.18^{* *}$ & $2.02 \pm 0.11$ & $5.08 \pm 0.66^{* *}$ \\
\hline $\mathrm{CM}$ & $0.05 \pm 0.01$ & $0.01 \pm 0.001^{* *}$ & $0.06 \pm 0.01$ & $0.04 \pm 0.02$ \\
\hline VLDL & $0.16 \pm 0.03$ & $0.09 \pm 0.004^{* * *}$ & $0.44 \pm 0.05$ & $0.49 \pm 0.25$ \\
\hline LDL & $0.71 \pm 0.06$ & $0.81 \pm 0.04$ & $0.31 \pm 0.02$ & $1.34 \pm 0.33^{*}$ \\
\hline $\mathrm{HDL}$ & $3.18 \pm 0.24$ & $4.66 \pm 0.15^{* *}$ & $1.21 \pm 0.04$ & $3.20 \pm 0.12^{* * *}$ \\
\hline
\end{tabular}

decrease in serum LPL activity is specific to wild-type mice. Statistical analyses also revealed that LPL activity in chow-fed ob/ $o b$ mice was lower than that in chow-fed wild-type mice $(P<0.01)$. However, LPL activity in LCKD-fed ob/ob mice was higher than that in LCKD-fed wild-type mice $(P<0.05)$.

\section{DISCUSSION}

A novel effect of the LCKD on induction of VLDLR expression was found in the liver, where this receptor is not normally expressed. Previous studies reported that the peroxisome proliferatoractivated receptor a agonist fenofibrate promotes not only hepatic VLDLR expression ${ }^{22}$ but also steatosis, ${ }^{23}$ which is in agreement with the phenotype of the LCKD-fed wild-type mice in the present study. The induction of hepatic VLDLR expression by fenofibrate or over-expression of VLDLR decreases triglyceride-rich VLDL levels via triglyceride clearance by the liver, ${ }^{22,24}$ which can promote the development of liver steatosis.

F3666 is a commonly used LCKD for rodents, and several recent studies have examined the effects of the low amounts of choline and protein in this diet. ${ }^{14,15} \mathrm{~A}$ lack of sufficient choline is associated with the development of steatosis in the liver, ${ }^{14,15,25}$ which impairs hepatic triglyceride secretion due to an absence of phosphatidylcholine synthesis. ${ }^{25}$ Choline supplementation can improve this pathologic condition. ${ }^{14,15}$ The low protein content of the F3666 diet increases the expression of several genes in the liver, but this effect is attenuated by methionine supplementation. ${ }^{15}$ These observations suggest that the low choline and protein content of the F3666 diet also played a role in the effects observed in our model. However, in ob/ob mice in our model, the F3666 diet prevented the progression of liver steatosis observed in chow-fed ob/ob mice (Fig. 1). Furthermore, the F3666 diet used in our study contained an abundance of choline-containing phospholipids (phosphatidylcholines and sphingomyelins, $5.8 \pm 0.2 \mathrm{~g}$ $\mathrm{kg}^{-1}$ ), and serum levels of choline-containing phospholipids in F3666-fed mice were also significantly higher than the levels in chow-fed mice (Supplementary Fig. S2). These results indicate that choline was supplied to peripheral tissues in sufficient amounts in 


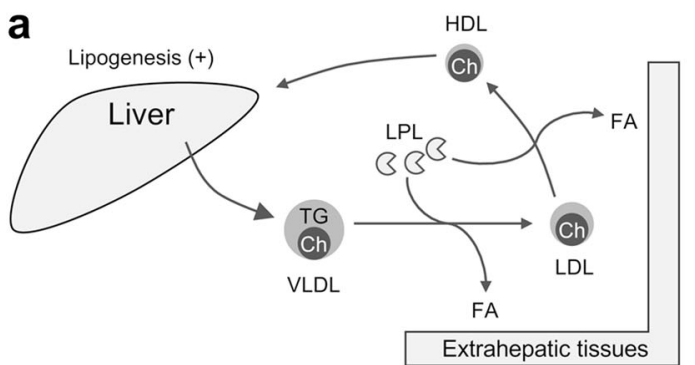

chow-fed wild-type mouse

b

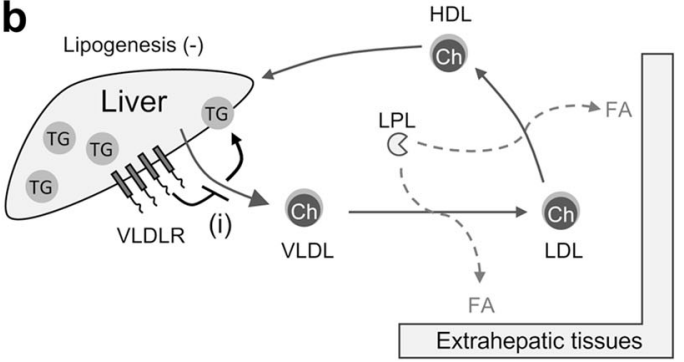

LCKD-fed wild-type mouse

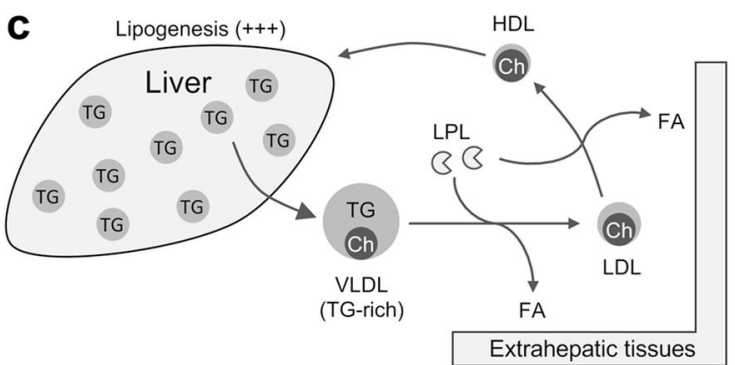

chow-fed ob/ob mouse

d

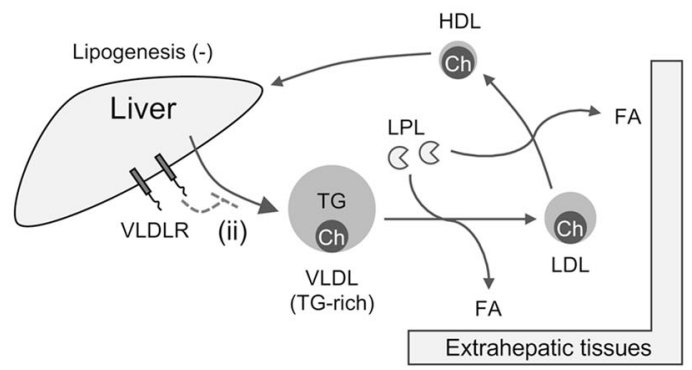

LCKD-fed ob/ob mouse

Fig. 3 Schematic illustrations of VLDL dynamics and metabolism in LCKD-fed mice. Overview of VLDL dynamics and metabolism in chow-fed wild-type $\mathbf{a}$, LCKD-fed wild-type mice $\mathbf{b}$, chow-fed $o b / o b$ mice $\mathbf{c}$, and LCKD-fed $o b / o b$ mice $\mathbf{d}$. The size and number of objects are drawn to reflect the experimental results. The working hypothesis regarding the effect of hepatic VLDLR on VLDL is as follows: (i) in wild-type mice, LCKD induces hepatic VLDLR expression, which inhibits triglyceride (TG) secretion via VLDL; (ii) in ob/ob mice, LCKD induces hepatic VLDLR expression, whereas the VLDLR does not function for TG-rich VLDL, which is specifically generated in the ob/ob strain. Ch, cholesterol; FA, fatty acid

the mice using this study, and therefore, choline levels had a minimal effect on development of steatosis.

We also observed VLDLR expression in the liver of LCKD-fed $o b / o b$ mice, in which the hyperphagic phenotype can compensate for the low protein content of the LCKD. This result indicates that the low protein content of the diet is not associated with increased VLDLR expression. As the low protein content of the F3666 diet plays an important role in the generation of ketone bodies in rodents, ${ }^{19}$ we conclude that the low-carbohydrate and high-fat/ketogenic properties of the LCKD induce hepatic VLDLR expression.

Based on the results of our present and previous studies, ${ }^{13,16}$ Fig. 3 illustrates the dynamics and metabolism of VLDL in LCKDfed mice and presents a working hypothesis of how VLDLR and LPL affect VLDL-triglyceride kinetics. In LCKD-fed wild-type mice, induction of hepatic VLDLR expression and decreased serum LPL activity inhibit the transport of triglycerides by VLDL from the liver to extrahepatic tissues, ultimately promoting steatosis (Fig. 3b). LCKD-induced VLDLR expression promotes triglyceride clearance from VLDL in the liver and the progression of liver steatosis. As LPL activity is necessary for the clearance of triglycerides from VLDL in peripheral tissues, ${ }^{18}$ decreased LPL activity slows triglyceride transport from the liver to peripheral tissues and promotes the development of liver steatosis. In LCKD-fed ob/ob mice, the generation of triglyceride-rich VLDL and maintained LPL activity promote the transport of triglycerides from the liver to extrahepatic tissues, ultimately leading to improvement of steatosis (Fig. 3d). Maintained LPL activity promotes the clearance of triglycerides from VLDL in peripheral tissues, which can accelerate triglyceride transport from the liver to peripheral tissues and contribute to the improvement of liver steatosis. LCKD-induced VLDLR expression has a minimal effect on triglyceride-rich VLDL, which is specifically generated in the $o b /$ $o b$ strain. LCKD-fed ob/ob mice became obese to the same degree as chow-fed ob/ob mice, in agreement with the observed effective transport of VLDL-triglycerides from the liver to extrahepatic tissues in these mice.

In ob/ob mice, LCKD feeding induces the production of triglyceride-rich VLDL, which has a long plasma residence time due to slow catabolism. $^{26,27}$ This property indicates that triglyceride-rich VLDL is readily delivered to extrahepatic tissues even though VLDLR expression is induced in the liver. As an absence of hepatic leptin signaling leads to elevated triglyceride levels in VLDL particles, ${ }^{28}$ the production of triglyceride-rich VLDL is likely associated with leptin deficiency in ob/ob mice. ${ }^{29}$ The maintenance of LPL activity in LCKD-fed ob/ob mice could also accelerate the release of triglycerides to extrahepatic tissues. As leptin regulates LPL production and activity in the tissues, ${ }^{30}$ maintenance of LPL activity is also likely associated with leptin deficiency in ob/ob mice. ${ }^{29}$

Based on these observations, we conclude that the transport of triglycerides via VLDL from the liver to extrahepatic tissues is inhibited by LCKD-induced hepatic VLDLR expression under conditions of low LPL activity. As this inhibition of triglyceride transport is rescued in leptin-deficient mice, regulating VLDL metabolism by hepatic VLDLR, LPL, and leptin may represent a new therapeutic strategy for preventing diet-induced liver steatosis.

\section{METHODS}

Animals and dietary studies

Dietary studies using female ob/ob and wild-type mice (B6.Cg-Lep ${ }^{\circ b} / \mathrm{J}$ and C57BL6J, Charles River Laboratories Japan, Yokohama, Japan) were conducted as reported previously. ${ }^{13}$ CE-2 (CLEA Japan, Tokyo, Japan), composed of $58.2 \%$ carbohydrate, $12.6 \%$ fat, and $29.2 \%$ protein by calories, was used as regular chow. F3666 (Bio-Serv, Frenchtown, NJ) was used as the LCKD, which is composed of $1.7 \%$ carbohydrate, $93.9 \%$ fat, and $4.4 \%$ protein by calories. Five-week-old mice were raised on either regular chow or the LCKD for 7 weeks. During this period, the blood glucose level was monitored at 3:00 p.m. on the first day of every week, and tissue samples were collected at the end of the dietary study. Blood glucose and $\beta$-hydroxybutyrate levels were determined using venous blood collected 
from the tail vein with a Precision Xceed Monitoring System (Abbott Laboratories, Abbott Park, IL, USA).

The Committee for Experiments Involving Animals of the National Institute of Advanced Industrial Science and Technology approved all animal experiments.

\section{Gene expression analysis}

Preparation of total RNA and gene expression analyses were conducted as reported previously. ${ }^{21}$ Agilent Expression Microarray analysis for gene expression profiling in tissues was conducted by Takara Bio (Shiga, Japan). The resulting microarray data were analyzed using the Aqua microarray viewer and Aqua $t$ test (Takara Bio) and deposited in the GEO under accession number GSE115342. Relative quantification of target gene expression by real-time PCR was performed using a Light Cycler ${ }^{\circledR} 480$ II system (Roche, Penzberg, Germany) with the following VIdlr gene-specific primers: forward, 5'-gcccgttctactcagtgtatcc-3'; reverse, 5'-gaactcatctgcactacatgttatgtt-3' (accession number of the VIdlr gene: NM_013703, GenBank). Reactions were performed using a KAPA SYBR ${ }^{\circledR}$ FAST qPCR Kit (KAPA Biosystems, Wilmington, MA) according to the manufacturer's instructions. As it is stably expressed in the liver, ${ }^{21}$ Eef1a1 was chosen as a housekeeping gene and used as the internal reference for subsequent realtime PCR analyses.

\section{Immunoblot analysis}

Protein extraction and immunoblot analyses were conducted according to a previously reported method ${ }^{16,31}$ using an anti-VLDLR antibody (AF2258; Bio-Techne, Minneapolis, MN, USA).

\section{Lipoprotein analysis}

The triglyceride and cholesterol profiles of serum lipoproteins were determined using a high-sensitivity lipoprotein profiling system employing high-performance liquid chromatography (Skylight Biotech Inc., Akita, Japan), as reported previously. ${ }^{13}$

\section{Measurement of LPL activity}

Serum LPL activity (units $\mathrm{ml}^{-1}$ ) was measured using an LPL Activity Assay Kit (Cell Biolabs, Inc., San Diego, CA, USA) according to the manufacturer's instructions.

Analysis of choline-containing phospholipids in the F3666 LCKD and serum

The content of choline-containing phospholipids was determined using a Phosphatidylcholine Assay Kit (Phospholipids C, FUJIFILM Wako Pure Chemical Corp., Osaka, Japan) according to the manufacturer's instructions.

\section{Statistical analysis}

After determination of variance using the $F$ test, statistical significance was evaluated using the two-tailed Student's $t$ test, with statistical significance defined as follows: ${ }^{*} P<0.05$, ${ }^{* *} P<0.01$, and ${ }^{* *} P<0.001$.

\section{Reporting summary}

Further information on research design is available in the Nature Research Reporting Summary linked to this article.

\section{DATA AVAILABILITY}

The datasets generated during and/or analyzed during the current study are available in the GEO repository under accession number GSE115342 or from the corresponding author on reasonable request.

Received: 30 May 2019; Accepted: 30 September 2019; Published online: 02 December 2019

\section{REFERENCES}

1. Gano, L. B., Patel, M. \& Rho, J. M. Ketogenic diets, mitochondria, and neurological diseases. J. Lipid Res. 55, 2211-2228 (2014).
2. Shai, l. et al. Weight loss with a low-carbohydrate, Mediterranean, or low-fat diet. N. Engl. J. Med. 359, 229-241 (2008).

3. Westman, E. C., Tondt, J., Maguire, E. \& Yancy, W. S. Jr. Implementing a lowcarbohydrate, ketogenic diet to manage type 2 diabetes mellitus. Expert Rev. Endocrinol. Metab. 13, 263-272 (2018).

4. Ruskin, D. N. et al. Ketogenic diet improves core symptoms of autism in BTBR mice. PLOS ONE 8, e65021 (2013).

5. Krebs, P. et al. Lethal mitochondrial cardiomyopathy in a hypomorphic Med30 mouse mutant is ameliorated by ketogenic diet. Proc. Natl. Acad. Sci. USA 108, 19678-19682 (2011).

6. Weber, D. D. et al. Ketogenic diet in the treatment of cancer-where do we stand? Mol. Metab. in press. https://www.sciencedirect.com/science/article/pii/ S2212877819304272?via\%3Dihub (2019).

7. Browning, J. D. et al. Short-term weight loss and hepatic triglyceride reduction: evidence of a metabolic advantage with dietary carbohydrate restriction. Am. J. Clin. Nutr. 93, 1048-1052 (2011).

8. Schugar, R. C. \& Crawford, P. A. Low-carbohydrate ketogenic diets, glucose homeostasis, and nonalcoholic fatty liver disease. Curr. Opin. Clin. Nutr. Metab. Care 15, 374-380 (2012).

9. Arslan, N. et al. Is ketogenic diet treatment hepatotoxic for children with intractable epilepsy? Seizure 43, 32-38 (2016).

10. Badman, M. K. et al. A very low carbohydrate ketogenic diet improves glucose tolerance in ob/ob mice independently of weight loss. Am. J. Physiol. Endocrinol. Metab. 297, E1197-E1204 (2009).

11. Jornayvaz, F. R. et al. A high-fat, ketogenic diet causes hepatic insulin resistance in mice, despite increasing energy expenditure and preventing weight gain. Am. J. Physiol. Endocrinol. Metab. 299, E808-E815 (2010).

12. Garbow, J. R. et al. Hepatic steatosis, inflammation, and ER stress in mice maintained long term on a very low-carbohydrate ketogenic diet. Am. J. Physiol. Gastrointest. Liver Physiol. 300, G956-G967 (2011).

13. Okuda, T. \& Morita, N. A very low carbohydrate ketogenic diet prevents the progression of hepatic steatosis caused by hyperglycemia in a juvenile obese mouse model. Nutr. Diabetes 2, e50 (2012).

14. Schugar, R. C. et al. Role of choline deficiency in the fatty liver phenotype of mice fed a low protein, very low carbohydrate ketogenic diet. PLOS ONE 8, e74806 (2013).

15. Pissios, $\mathrm{P}$. et al. Methionine and choline regulate the metabolic phenotype of a ketogenic diet. Mol. Metab. 2, 306-313 (2013).

16. Okuda, T. \& Morita, N. A very low carbohydrate ketogenic diet increases hepatic glycosphingolipids related to regulation of insulin signaling. J. Funct. Foods $\mathbf{2 1}$ 70-74 (2016).

17. Takahashi, S. et al. The very low-density lipoprotein (VLDL) receptor: characterization and functions as a peripheral lipoprotein receptor. J. Atheroscler. Thromb. 11, 200-208 (2004)

18. Kawano, Y. \& Cohen, D. E. Mechanisms of hepatic triglyceride accumulation in non-alcoholic fatty liver disease. J. Gastroenterol. 48, 434-441 (2013).

19. Bough, K. J. \& Eagles, D. A. A ketogenic diet increases the resistance to pentylenetetrazole-induced seizures in the rat. Epilepsia 40, 138-143 (1999)

20. Coleman, D. L. \& Hummel, K. P. The influence of genetic background on the expression of the obese $(\mathrm{Ob})$ gene in the mouse. Diabetologia 9, 287-293 (1973).

21. Okuda, T. A low-carbohydrate ketogenic diet promotes ganglioside synthesis via the transcriptional regulation of ganglioside metabolism-related genes. Sci. Rep. 9, 7627 (2019).

22. Gao, Y. et al. Upregulation of hepatic VLDLR via PPARa is required for the triglyceride-lowering effect of fenofibrate. J. Lipid Res. 55, 1622-1633 (2014).

23. Yan, F. et al. Peroxisome proliferator-activated receptor a activation induces hepatic steatosis, suggesting an adverse effect. PLoS ONE 9, e99245 (2014).

24. Tacken, P. J. et al. LDL receptor deficiency unmasks altered VLDL triglyceride metabolism in VLDL receptor transgenic and knockout mice. J. Lipid Res. 41 2055-2062 (2000).

25. Corbin, K. D. \& Zeisel, S. H. Choline metabolism provides novel insights into nonalcoholic fatty liver disease and its progression. Curr. Opin. Gastroenterol. 28, 159-165 (2012).

26. Packard, C. J. \& Shepherd, J. Lipoprotein heterogeneity and apolipoprotein B metabolism. Arterioscler. Thromb. Vasc. Biol. 17, 3542-3556 (1997).

27. Demant, T. \& Packard, C. In vivo studies of VLDL metabolism and LDL heterogeneity. Eur. Heart J. 19(Suppl. H), H7-H10 (1998).

28. Huynh, F. K. et al. A role for hepatic leptin signaling in lipid metabolism via altered very low density lipoprotein composition and liver lipase activity in mice. Hepatology 57, 543-554 (2013).

29. Zhang, Y. et al. Positional cloning of the mouse obese gene and its human homologue. Nature 372, 425-432 (1994). 
30. Donahoo, W. T. et al. Leptin increases skeletal muscle lipoprotein lipase and postprandial lipid metabolism in mice. Metabolism 60, 438-443 (2011).

31. Okuda, T., Fukui, A. \& Morita, N. Altered expression of O-GlcNAc-modified proteins in a mouse model whose glycemic status is controlled by a low carbohydrate ketogenic diet. Glycoconj. J. 30, 781-789 (2013).

\section{ACKNOWLEDGEMENTS}

This work was supported by JSPS KAKENHI Grant Number 15H02907 and 19K11810. We thank A. Fukui and A. Kato for technical assistance.

\section{AUTHOR CONTRIBUTIONS}

T.O. conceived, designed, performed, analyzed the experiments, and wrote the paper.

\section{COMPETING INTERESTS}

The author declares no competing interests.

\section{ADDITIONAL INFORMATION}

Supplementary information is available for this paper at https://doi.org/10.1038/ s41538-019-0058-4.
Correspondence and requests for materials should be addressed to T.O.

Reprints and permission information is available at http://www.nature.com/ reprints

Publisher's note Springer Nature remains neutral with regard to jurisdictional claims in published maps and institutional affiliations.

cc) (i)

Open Access This article is licensed under a Creative Commons Attribution 4.0 International License, which permits use, sharing, adaptation, distribution and reproduction in any medium or format, as long as you give appropriate credit to the original author(s) and the source, provide a link to the Creative Commons license, and indicate if changes were made. The images or other third party material in this article are included in the article's Creative Commons license, unless indicated otherwise in a credit line to the material. If material is not included in the article's Creative Commons license and your intended use is not permitted by statutory regulation or exceeds the permitted use, you will need to obtain permission directly from the copyright holder. To view a copy of this license, visit http://creativecommons. org/licenses/by/4.0/.

(c) The Author(s) 2019 